\title{
Detecting Multiparticle Entanglement of Dicke States
}

\author{
Bernd Lücke, ${ }^{1}$ Jan Peise, ${ }^{1}$ Giuseppe Vitagliano, ${ }^{2}$ Jan Arlt,${ }^{3}$ Luis Santos, ${ }^{4}$ Géza Tóth, ${ }^{2,5,6}$ and Carsten Klempt ${ }^{1}$ \\ ${ }^{1}$ Institut für Quantenoptik, Leibniz Universität Hannover, Welfengarten 1, D-30167 Hannover, Germany \\ ${ }^{2}$ Department of Theoretical Physics, University of the Basque Country UPV/EHU, P.O. Box 644, E-48080 Bilbao, Spain \\ ${ }^{3}$ QUANTOP, Institut for Fysik og Astronomi, Aarhus Universitet, 8000 Århus C, Denmark \\ ${ }^{4}$ Institut für Theoretische Physik, Leibniz. Universität Hannover, Appelstraße 2, D-30167 Hannover, Germany \\ ${ }^{5}$ IKERBASQUE, Basque Foundation for Science, E-48011 Bilbao, Spain \\ ${ }^{6}$ Wigner Research Centre for Physics, Hungarian Academy of Sciences, P.O. Box 49, H-1525 Budapest, Hungary
}

(Received 27 February 2014; published 17 April 2014)

\begin{abstract}
Recent experiments demonstrate the production of many thousands of neutral atoms entangled in their spin degrees of freedom. We present a criterion for estimating the amount of entanglement based on a measurement of the global spin. It outperforms previous criteria and applies to a wider class of entangled states, including Dicke states. Experimentally, we produce a Dicke-like state using spin dynamics in a Bose-Einstein condensate. Our criterion proves that it contains at least genuine 28-particle entanglement. We infer a generalized squeezing parameter of $-11.4(5) \mathrm{dB}$.
\end{abstract}

DOI: 10.1103/PhysRevLett.112.155304

PACS numbers: 67.85.-d, 03.67.Bg, 03.67.Mn, 03.75.Mn

Entanglement, one of the most intriguing features of quantum mechanics, is nowadays a key ingredient for many applications in quantum information science [1,2], quantum simulation [3,4], and quantum-enhanced metrology [5]. Entangled states with a large number of particles cannot be characterized via full state tomography [6], which is routinely used in the case of photons $[7,8]$, trapped ions [9], or superconducting circuits [10,11]. A reconstruction of the full density matrix is hindered and finally prevented by the exponential increase of the required number of measurements. Furthermore, it is technically impossible to address all individual particles or even fundamentally forbidden if the particles occupy the same quantum state. Therefore, the entanglement of manyparticle states is best characterized by measuring the expectation values and variances of the components of the collective spin $\mathbf{J}=\left(J_{x}, J_{y}, J_{z}\right)^{T}=\sum_{i} \mathbf{s}_{i}$, the sum of all individual spins $\mathbf{s}_{i}$ in the ensemble.

In particular, the spin-squeezing parameter $\xi^{2}=$ $N\left(\Delta J_{z}\right)^{2} /\left(\left\langle J_{x}\right\rangle^{2}+\left\langle J_{y}\right\rangle^{2}\right)$ defines the class of spinsqueezed states for $\xi^{2}<1$. This inequality can be used to verify the presence of entanglement, since all spinsqueezed states are entangled [12]. Large clouds of entangled neutral atoms are typically prepared in such spin-squeezed states, as shown in thermal gas cells [13], at ultracold temperatures [14-16], and in Bose-Einstein condensates [17-19].

Systems with multiple particles may exhibit more than pairwise entanglement. Multiparticle entanglement is best

Published by the American Physical Society under the terms of the Creative Commons Attribution 3.0 License. Further distribution of this work must maintain attribution to the author(s) and the published article's title, journal citation, and DOI. quantified by means of the so-called entanglement depth, defined as the number of particles in the largest nonseparable subset [see Fig. 1(a)]. There have been numerous experiments detecting multiparticle entanglement involving up to 14 qubits in systems, where the particles can be addressed individually [9,20-24]. Large ensembles of neutral atoms
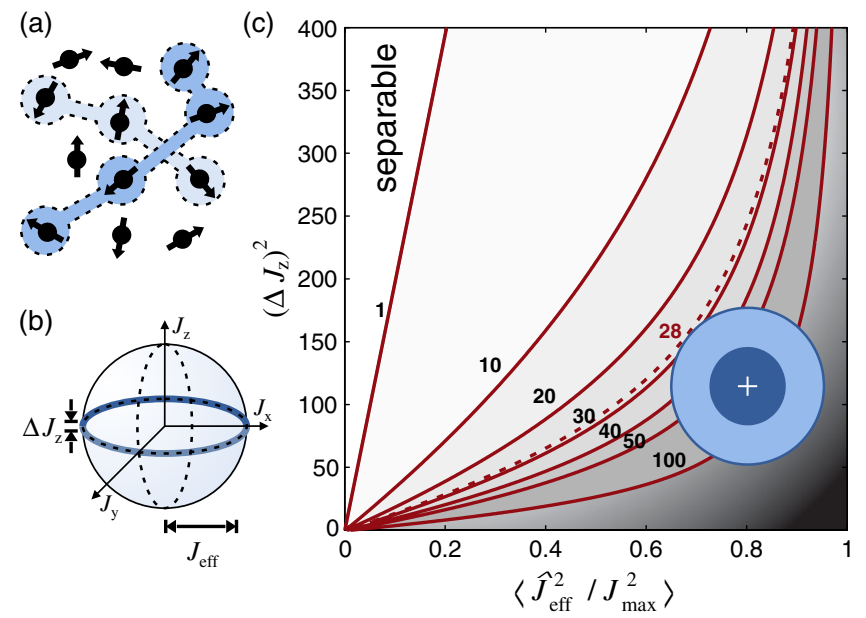

FIG. 1 (color online). Measurement of the entanglement depth for a total number of 8000 atoms. (a) The entanglement depth is given by the number of atoms in the largest nonseparable subset (shaded areas). (b) The spins of the individual atoms add up to the total spin $\mathbf{J}$ whose possible orientations can be depicted on the Bloch sphere. Dicke states are represented by a ring around the equator with an ultralow width $\Delta J_{z}$ and a large radius $J_{\text {eff }}$. (c) The entanglement depth in the vicinity of a Dicke state can be inferred from a measurement of these values. The red lines indicate the boundaries for various entanglement depths. The experimental result is shown as blue uncertainty ellipses with 1 and 2 standard deviations, proving an entanglement depth larger than 28 (dashed line). 
pose the additional challenge of obtaining the entanglement depth from collective measurements. Following the criterion for $k$-particle entanglement of Ref. [25], multiparticle entanglement has been experimentally demonstrated in spin-squeezed Bose-Einstein condensates [17]. However, the method only applies to spin-squeezed states, which constitute a small subset of all possible entangled manyparticle states. Moreover, the strong entanglement of states with extreme sub-shot-noise fluctuations is not detected under the influence of minimal experimental noise (see the Supplemental Material [26]). Whereas entanglement detection for more general entangled states has already been developed [31,32], it is desirable to extend these methods towards the detection of multiparticle entanglement.

In this Letter, we introduce a method for the quantification of entanglement. Our criterion is applicable to both spin-squeezed and more extreme states, yielding superior results compared to the inspiring work by Sørensen and Mølmer [25] and Duan [33]. It enables us to quantify the multiparticle entanglement of an experimentally created Dicke-like state, yielding a minimum entanglement depth of 28. In addition, we extract a generalized squeezing parameter, which is also applicable to Dicke states of $-11.4(5) \mathrm{dB}$, which is so far the best reported value in any atomic system.

Dicke states [34] constitute a particularly relevant class of highly entangled, but not spin-squeezed, states. They are simultaneous eigenstates $|J, M\rangle$ of $\mathbf{J}^{2}$ and $J_{z}$, and the spin-squeezing parameter $\xi^{2}$ does not detect them as entangled [35]. Nonetheless, Dicke states have optimal metrological properties [36-38] and can be used to reach Heisenberg-limited sensitivity [39]. They are also useful for quantum information processing tasks, such as $1 \rightarrow(N-1)$ telecloning or open-destination teleportation [40]. Experimentally, high-fidelity Dicke states with small particle numbers have been created with photons [22,23] and trapped ions [9], and have been detected by global measurements [41].

Among other methods [42,43], large numbers of atoms in Dicke states with $|J, M=0\rangle$ may be created in spinor Bose-Einstein condensates [44]. Spin dynamics creates a superposition of Dicke states with a varying total number of particles in a process that resembles optical parametric down-conversion [45,46]. In previous work, the entanglement of these states was proven by a homodyne measurement [47] and by a test of the metrological sensitivity beyond shot noise [48]. However, the achieved metrological sensitivity did not imply more than pairwise entanglement [38].

For the generation of the desired Dicke states, we prepare a ${ }^{87} \mathrm{Rb}$ Bose-Einstein condensate of $2 \times 10^{4}$ atoms in a crossed-beam dipole trap with trapping frequencies of $2 \pi \times(200,150,150) \mathrm{Hz}$. Initially prepared in the Zeeman level $\left(F, m_{F}\right)=(1,0)$, atoms collide and form correlated pairs in the two Zeeman levels $(1, \pm 1)$. These atoms are
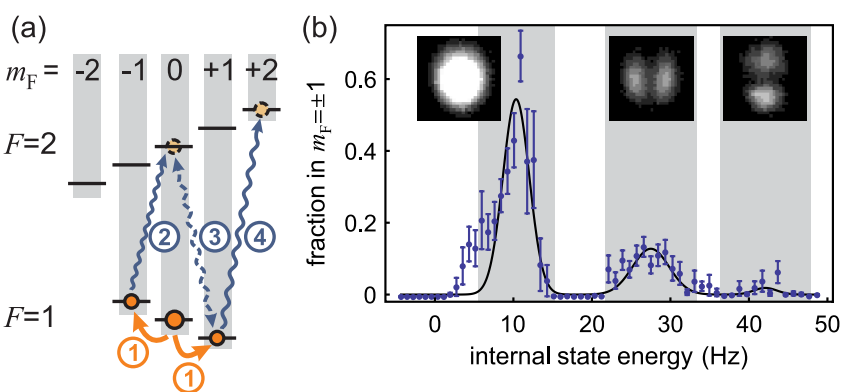

FIG. 2 (color online). Preparation and detection of a Dicke-like state. (a) A Bose-Einstein condensate in the level $\left(F, m_{F}\right)=$ $(1,0)$ generates clouds with the same number of atoms in the levels $(1, \pm 1)(1)$. A microwave pulse (2) transfers the atoms from $(1,-1)$ to $(2,0)$. Optionally, a microwave pulse (3) can be used to couple the two clouds for the measurement of $J_{\text {eff }}$. Finally, the atoms in the level $(1,1)$ are transferred to $(2,2)$ before detection. (b) The number of atoms is measured by standard absorption imaging (insets). On well-resolved resonances depending on the internal state energy, distinct spatial modes are populated with a large fraction of the total number of atoms. The black line is a Gaussian fit to guide the eye. In our experiments, we use the resonance at $\approx 28 \mathrm{~Hz}$.

transferred to distinct spatial modes [45,49], which are addressed by microwave dressing [44] the Zeeman level $(1,1)$ [Fig. 2(b)]. In an experimental run, up to $N=8 \times$ $10^{3}$ atoms are transferred to the first excited mode along the strongest trap axis within $240 \mathrm{~ms}$. Since they are transferred pairwise, we expect an equal number of atoms $N_{ \pm 1}=$ $(N / 2)$ in the two Zeeman levels $(1, \pm 1)$. These atoms are highly entangled in analogy to optical parametric downconversion. It is the central objective of this Letter to quantify the entanglement depth of the created manyparticle state.

We restrict the description of the output state to the two relevant Zeeman levels $(1, \pm 1)$. In this pseudo-spin- $1 / 2$ system, we characterize the state by the collective spin $\mathbf{J}$, resulting from the sum of the individual pseudospins. In this picture, the ideal output state with an equal number of atoms constitutes the Dicke state $|J=(N / 2), M=0\rangle$ with vanishing fluctuations $\Delta J_{z}$. The fluctuations of the collective spin can be measured directly by counting the number of atoms in the two Zeeman levels. For this purpose, we transfer the atoms to the levels $(2,0)$ and $(2,2)$ with microwave pulses [see Fig. 2(a)]. Subsequently, the trap is switched off and a strong magnetic field gradient separates the spin components during ballistic expansion. The number of atoms is then measured by standard absorption imaging. The absolute number of atoms was calibrated [48] and it was confirmed that shot noise fluctuations are observed for a coherent state [see Fig. 3(a)], which was created by splitting a Bose-Einstein condensate with a $\pi / 2$ microwave pulse.

We measure $J_{x}$ and $J_{y}$ by rotating the total spin using a $\pi / 2$ microwave coupling pulse on the $(1,1)$ to $(2,0)$ 
(a)

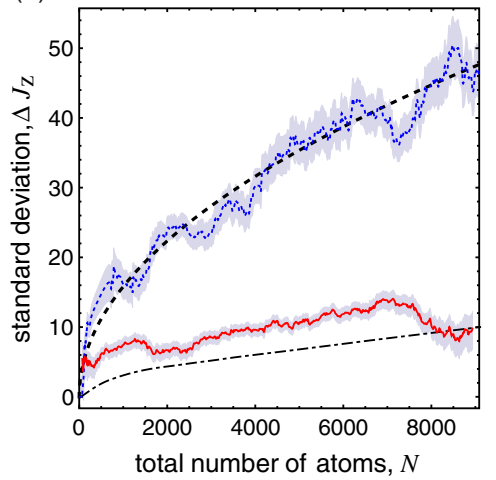

(b)

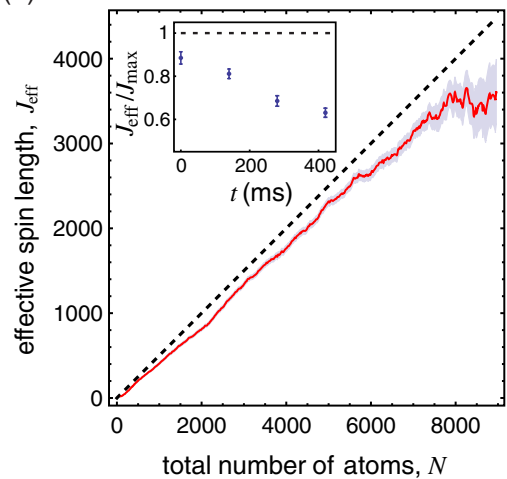

(c)

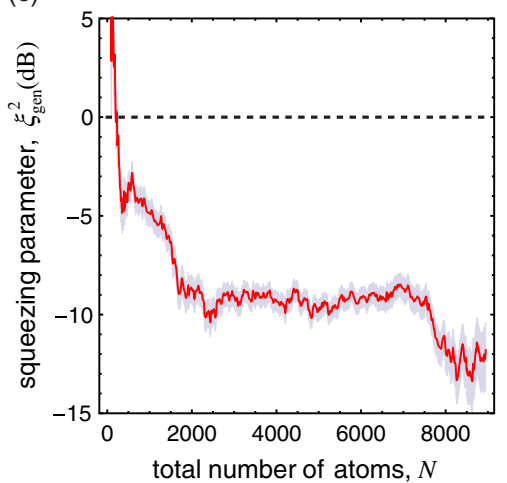

FIG. 3 (color online). Characterization of the experimentally created Dicke-like state. (a) Measurement of the width $\Delta J_{z}$ for varying the total number of atoms (red solid line). Each value and its statistical uncertainty (gray shading) is calculated for a 1000-atom interval within the total number of atoms. The measured values of $\Delta J_{z}$ are well below the shot noise limit (theory: black dashed line, experiment: blue dotted line) and partially explained by a lower limit of the number-dependent detection noise (black dash-dotted line). (b) The measured value of $J_{\text {eff }}$ as a function of the total number of atoms almost reaches its optimal value (black dashed line). The inset shows that the normalized $J_{\text {eff }}$ is slowly reduced during an additional hold time. (c) The recorded data allow for a determination of the generalized squeezing parameter as a function of the total number of atoms. At a total of 8000 atoms, it reaches a value of $-11.4(5) \mathrm{dB}$.

transition before the number measurement [see Fig. 2(a)]. Whether $J_{x}$ or $J_{y}$ is measured depends on the relation between the microwave phase and the phase of the initial Bose-Einstein condensate. The condensate phase represents the only possible phase reference in analogy to the local oscillator in optics. Intrinsically, it has no relation to the microwave phase, such that we homogeneously average over all possible phase relations in our measurements. For a given phase difference $\alpha$, a rotation yields a measurement of $J_{\alpha} \equiv \cos \alpha J_{x}+\sin \alpha J_{y}$. Averaging over all possible $\alpha$, the measured expectation value of the second moment corresponds to $(1 / 2 \pi) \int_{0}^{2 \pi}\left\langle J_{\alpha}^{2}\right\rangle \mathrm{d} \alpha=\left\langle(1 / 2)\left(J_{x}^{2}+J_{y}^{2}\right)\right\rangle$. After a random rotation, we thus record the effective spin length $J_{\text {eff }}^{2}=\left\langle\hat{J}_{\text {eff }}^{2}\right\rangle=\left\langle J_{x}^{2}+J_{y}^{2}\right\rangle$, which equals the spin length in the limit of vanishing $\left\langle J_{z}^{2}\right\rangle[50]$. Dicke states can be ideally characterized by the measurement of a large $J_{\text {eff }}$ and a small variance $\left(\Delta J_{z}\right)^{2}$ [see Fig. 1(b)].

Figure 3(a) depicts the results of our measurement of $\Delta J_{z}$ depending on the total number of atoms $N$. The recorded fluctuations were corrected for the independently measured detection noise of 10.9(3) atoms to obtain the pure atomic noise. The detection noise was directly extracted from images of the detection beams and is mainly caused by the photoelectron shot noise on the camera. The measured atom number fluctuations are well below the atomic shot noise level, reaching down to $-12.4 \pm 1.2 \mathrm{~dB}$ at a total number of 8000 atoms. The fluctuations are almost independent of the total number of atoms with a small trend of $0.15 \sqrt{N}$. We do not record an increase of the measured fluctuations for a variable additional hold time of up to $420 \mathrm{~ms}$. Thus, we can exclude three-body losses, collisions with the background gas or radio-frequency noise as relevant noise sources. We attribute the measured fluctuations to an additional detection noise since photoelectron shot noise and the influence of technical noise of the imaging beams are expected to increase slightly for a larger number of atoms. The solid line in Fig. 3(a) shows an estimated lower limit of this effect [26].

A measurement of the effective spin length $J_{\text {eff }}$ is presented in Fig. 3(b). The values for $J_{\text {eff }}$ almost reach their optimal value of $J_{\max }=N / 2$. This measurement shows that the created state is nearly fully symmetric. After a variable hold time, the measured effective spin length diminishes slowly [see Fig. 3(b) inset]. We thus conclude that the measurement result is limited by magnetic field gradients and collisions. Elastic collisions can transfer individual atoms to other spatial modes, reducing the ensemble's purity and the achievable effective spin length. The combined measurements of $\Delta J_{z}$ and $J_{\text {eff }}$ prove that the created many-particle state is in the close vicinity of an ideal symmetric Dicke state.

The measurements can be combined to extract a generalized squeezing parameter $\xi_{\text {gen }}^{2}=(N-1)\left[\left(\Delta J_{z}\right)^{2} /\left\langle J_{x}^{2}\right\rangle+\right.$ $\left.\left\langle J_{y}^{2}\right\rangle-N / 2\right]$ which extends the concept of the spin-squeezing parameter to more general entangled states, including Dicke states [51-53]. Figure 3(c) presents the measured generalized squeezing parameter as a function of the total number of atoms. Note that the quasiconstant plateau is not statistically significant. At a total of $N=8000$ atoms, it reaches a value of $-11.4(5) \mathrm{dB}$. This represents the best reported value reached in any atomic system.

In addition to this proof of entanglement, the measured data allow for a quantification of the entanglement depth. Given states with an entanglement depth $k$, it is possible to deduce a minimal achievable $\left(\Delta J_{z}\right)^{2}$ for each value of $J_{\text {eff }}^{2}$ [26]. All states below this minimum must have an entanglement depth larger than $k$. It can be shown that the states on this boundary $|\Psi\rangle=|\psi\rangle^{\otimes(N / k)}$ 


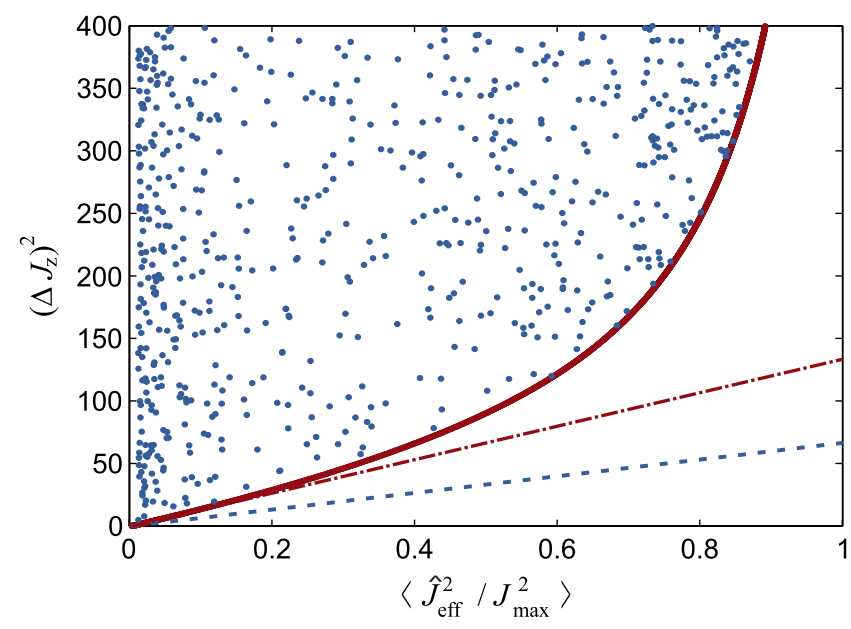

FIG. 4 (color online). Detection of $k$-particle entanglement based on the total spin. The red solid line marks the boundary for $k$-particle entangled states with $N=8000$ and $k=28$ in the $\left(\left\langle\hat{J}_{\text {eff }} / J_{\max }^{2}\right\rangle,\left(\Delta J_{z}\right)^{2}\right)$ plane. As a cross-check, random states with $k$-particle entanglement are plotted as blue dots, filling up the allowed region. The criterion of Ref. [33] only detects states that correspond to points below the dashed blue line. An improved linear criterion is gained from calculating a tangent to the new boundary (dash-dotted red line).

are tensor products of identical $k$-particle states $|\psi\rangle$. Interestingly, these $k$-particle states are ordinary spinsqueezed states. Figure 4 shows the boundary in the case of 28-particle entanglement at a total number of 8000 atoms. As a cross-check, random states with 28particle entanglement are plotted in the figure. This confirms that our criterion is optimal and superior to the linear condition of Ref. [33]. Finally, the criterion detects a larger entanglement depth than the criterion given in Ref. [25] when it is applied to spin-squeezed states with minimal experimental noise [26]. It thus outperforms the original criterion in experimentally realistic situations. Beyond spin-squeezing, the criterion is applicable to unpolarized states and thus allows for an optimal evaluation of the entanglement depth of a Dicke-like state as created in our experiments.

Figure 1(c) shows the entanglement depth of the created state for 8000 atoms. The red lines present the newly derived boundaries for $k$-particle entanglement. All separable (unentangled) states are restricted to the far left of the diagram, as indicated by the $k=1$ line. The measured values of $\left(\Delta J_{z}\right)^{2}$ and $\left\langle\hat{J}_{\text {eff }}^{2} / J_{\max }^{2}\right\rangle$ are represented by uncertainty ellipses with 1 and 2 standard deviations. The center of the ellipses corresponds to an entanglement depth of 68. With the confidence of 2 standard deviations, the data prove that our state has an entanglement depth larger than 28 . These numbers are only partly limited by the prepared state itself, but also by the number-dependent detection noise. This detection noise results in a larger measured value of $J_{z}^{2}$ and thus decreases the lower bound for the entanglement depth. This is the largest reported entanglement depth of Dicke-like states. In the future, the measured entanglement depth can be increased by an improved number detection, compensated magnetic field gradients and a faster spin dynamics.

In summary, we have presented a criterion for the detection of multiparticle entanglement based on a measurement of the ensemble's total spin. In the case of spin-squeezed states, the criterion outperforms the results of previous criteria in experimentally realistic situations. It also extends to more general entangled states, most importantly to Dicke states. We have applied the criterion to detect an entanglement depth larger than 28 in an experimentally created Dicke-like state. The experimental results also allow for a determination of a generalized squeezing parameter of $-11.4(5) \mathrm{dB}$.

We thank W. Ertmer, A. Smerzi, L. Pezzé, and P. Hyllus for inspiring discussions. We acknowledge support from the Centre QUEST and from the DFG (Research Training Group 1729). We also thank the DFF and the Lundbeck Foundation for support. We acknowledge support from the EMRP, which is jointly funded by the EMRP participating countries within EURAMET and the European Union. We thank the EU (ERC StG GEDENTQOPT, CHIST-ERA QUASAR), the MINECO (Project No. FIS2012-36673-C03-03), the Basque Government (Project No. IT4720-10), and the OTKA (Contract No. K83858).

[1] R. Horodecki, P. Horodecki, M. Horodecki, and K. Horodecki, Rev. Mod. Phys. 81, 865 (2009).

[2] O. Gühne and G. Tóth, Phys. Rep. 474, 1 (2009).

[3] K. Kim, M.-S. Chang, S. Korenblit, R. Islam, E. Edwards, J. Freericks, G.-D. Lin, L.-M. Duan, and C. Monroe, Nature (London) 465, 590 (2010).

[4] J. Simon, W. S. Bakr, R. Ma, M. E. Tai, P. M. Preiss, and M. Greiner, Nature (London) 472, 307 (2011).

[5] V. Giovannetti, S. Lloyd, and L. Maccone, Nat. Photonics 5, 222 (2011).

[6] Quantum State Estimation, edited by M. Paris and J. Řeháček, Lecture Notes in Physics, Vol. 649 (Springer, Berling, 2004).

[7] A. G. White, D. F. V. James, P. H. Eberhard, and P. G. Kwiat, Phys. Rev. Lett. 83, 3103 (1999).

[8] C. Schwemmer, G. Tóth, A. Niggebaum, T. Moroder, D. Gross, O. Gühne, and H. Weinfurter, arXiv:1401.7526.

[9] H. Häffner, W. Hänsel, C. F. Roos, J. Benhelm, D. Chek-al kar, M. Chwalla, T. Körber, U. D. Rapol, M. Riebe, P. O. Schmidt, C. Becher, O. Gühne, W. Dür, and R. Blatt, Nature (London) 438, 643 (2005).

[10] M. Neeley, R. C. Bialczak, M. Lenander, E. Lucero, M. Mariantoni, A. D. O' Connell, D. Sank, H. Wang, M. Weides, J. Wenner, Y. Yin, T. Yamamoto, A. N. Cleland, and J. M. Martinis, Nature (London) 467, 570 (2010).

[11] L. DiCarlo, M. Reed, L. Sun, B. Johnson, J. Chow, J. Gambetta, L. Frunzio, S. Girvin, M. Devoret, and R. Schoelkopf, Nature (London) 467, 574 (2010). 
[12] A. Sørensen, L.-M. Duan, J. I. Cirac, and P. Zoller, Nature (London) 409, 63 (2001).

[13] J. Hald, J. L. Sørensen, C. Schori, and E. S. Polzik, Phys. Rev. Lett. 83, 1319 (1999).

[14] M. H. Schleier-Smith, I. D. Leroux, and V. Vuletić, Phys. Rev. Lett. 104, 073604 (2010).

[15] Z. Chen, J. G. Bohnet, S. R. Sankar, J. Dai, and J. K. Thompson, Phys. Rev. Lett. 106, 133601 (2011).

[16] R. J. Sewell, M. Koschorreck, M. Napolitano, B. Dubost, N. Behbood, and M. W. Mitchell, Phys. Rev. Lett. 109, 253605 (2012).

[17] C. Gross, T. Zibold, E. Nicklas, J. Estève, and M. K. Oberthaler, Nature (London) 464, 1165 (2010).

[18] M. Riedel, P. Böhi, Y. Li, T. Hänsch, A. Sinatra, and P. Treutlein, Nature (London) 464, 1170 (2010).

[19] C. D. Hamley, C. S. Gerving, T. M. Hoang, E. M. Bookjans, and M. S. Chapman, Nat. Phys. 8, 305 (2012).

[20] C. A. Sackett, D. Kielpinski, B. E. King, C. Langer, V. Meyer, C. J. Myatt, M. Rowe, Q. A. Turchette, W. M. Itano, D. J. Wineland, and C. Monroe, Nature (London) 404, 256 (2000).

[21] T. Monz, P. Schindler, J. T. Barreiro, M. Chwalla, D. Nigg, W. A. Coish, M. Harlander, W. Hänsel, M. Hennrich, and R. Blatt , Phys. Rev. Lett. 106, 130506 (2011).

[22] W. Wieczorek, R. Krischek, N. Kiesel, P. Michelberger, G. Tóth, and H. Weinfurter, Phys. Rev. Lett. 103, 020504 (2009).

[23] R. Prevedel, G. Cronenberg, M. S. Tame, M. Paternostro, P. Walther, M. S. Kim, and A. Zeilinger, Phys. Rev. Lett. 103, 020503 (2009).

[24] W.-B. Gao, C.-Y. Lu, X.-C. Yao, P. Xu, O. Gühne, A. Goebel, Y.-A. Chen, C.-Z. Peng, Z.-B. Chen, and J.-W. Pan, Nat. Phys. 6, 331 (2010).

[25] A. S. Sørensen and K. Mølmer, Phys. Rev. Lett. 86, 4431 (2001).

[26] See Supplemental Material http://link.aps.org/supplemental/ 10.1103/PhysRevLett.112.155304, which includes Refs. [27-30].

[27] A. Stuart and K. Ord, Kendall's Advanced Theory of Statistics (Halsted Press, London, 1994), 6th ed.

[28] O. Gühne, G. Tóth, and H. J. Briegel, New J. Phys. 7, 229 (2005).

[29] P. Hyllus, L. Pezzé, A. Smerzi, and G. Tóth , Phys. Rev. A 86, 012337 (2012).

[30] G. Vitagliano and G. Tóth (unpublished).

[31] G. Tóth, C. Knapp, O. Gühne, and H. J. Briegel, Phys. Rev. Lett. 99, 250405 (2007).

[32] J. Tura, R. Augusiak, A. B. Sainz, T. Vértesi, M. Lewenstein, and A. Acín, arXiv:1306.6860.

[33] L.-M. Duan, Phys. Rev. Lett. 107, 180502 (2011).
[34] R. H. Dicke, Phys. Rev. 93, 99 (1954).

[35] J. Ma, X. Wang, C. Sun, and F. Nori , Phys. Rep. 509, 89 (2011).

[36] R. Krischek, C. Schwemmer, W. Wieczorek, H. Weinfurter, P. Hyllus, L. Pezzé, and A. Smerzi, Phys. Rev. Lett. 107, 080504 (2011).

[37] G. Tóth, Phys. Rev. A 85, 022322 (2012).

[38] P. Hyllus, W. Laskowski, R. Krischek, C. Schwemmer, W. Wieczorek, H. Weinfurter, L. Pezzé, and A. Smerzi, Phys. Rev. A 85, 022321 (2012).

[39] M. J. Holland and K. Burnett, Phys. Rev. Lett. 71, 1355 (1993).

[40] A. Chiuri, C. Greganti, M. Paternostro, G. Vallone, and P. Mataloni, Phys. Rev. Lett. 109, 173604 (2012).

[41] A. Noguchi, K. Toyoda, and S. Urabe, Phys. Rev. Lett. 109, 260502 (2012).

[42] T. Vanderbruggen, S. Bernon, A. Bertoldi, A. Landragin, and P. Bouyer, Phys. Rev. A 83, 013821 (2011).

[43] R. Bucker, J. Grond, S. Manz, T. Berrada, T. Betz, C. Koller, U. Hohenester, T. Schumm, A. Perrin, and J. Schmiedmayer, Nat. Phys. 7, 608 (2011).

[44] D. Stamper-Kurn and M. Ueda, Rev. Mod. Phys. 85, 1191 (2013).

[45] C. Klempt, O. Topic, G. Gebreyesus, M. Scherer, T. Henninger, P. Hyllus, W. Ertmer, L. Santos, and J. J. Arlt, Phys. Rev. Lett. 103, 195302 (2009).

[46] C. Klempt, O. Topic, G. Gebreyesus, M. Scherer, T. Henninger, P. Hyllus, W. Ertmer, L. Santos, and J. J. Arlt, Phys. Rev. Lett. 104, 195303 (2010).

[47] C. Gross, H. Strobel, E. Nicklas, T. Zibold, N. Bar-Gill, G. Kurizki, and M. K. Oberthaler, Nature (London) 480, 219 (2011).

[48] B. Lücke, M. Scherer, J. Kruse, L. Pezzé, F. Deuretzbacher, P. Hyllus, O. Topic, J. Peise, W. Ertmer, J. Arlt, L. Santos, A. Smerzi, and C. Klempt, Science 334, 773 (2011).

[49] M. Scherer, B. Lücke, G. Gebreyesus, O. Topic, F. Deuretzbacher, W. Ertmer, L. Santos, J. J. Arlt, and C. Klempt, Phys. Rev. Lett. 105, 135302 (2010).

[50] For small particle numbers, $J_{\text {eff }}$ is defined as $J_{\text {eff }}\left(J_{\text {eff }}+\right.$ $1)=\left\langle J_{x}^{2}+J_{y}^{2}\right\rangle$ with $0 \leq J_{\text {eff }} \leq(N / 2)$. For large particle numbers, we approximate $J_{\text {eff }}^{2} \approx\left\langle J_{x}^{2}+J_{y}^{2}\right\rangle$.

[51] G. Vitagliano, P. Hyllus, I. L. Egusquiza, and G. Tóth, Phys. Rev. Lett. 107, 240502 (2011).

[52] G. Vitagliano, I. Apellaniz, I. L. Egusquiza, and G. Tóth, Phys. Rev. A 89, 032307 (2014).

[53] The new parameter has been used to study the dynamics of the modified Lipkin-Meshkov-Glick model in M. A. Marchiolli, D. Galetti, and T. Debarba, Int. J. Quantum. Inform. 11, 1330001 (2013). 